\title{
La musique dans la vie des barbares et des Grecs qui habitaient le littoral septentional de la Mer Noire
}

\author{
I. Vdovichenko and N. Khlebko \\ Ukraine
}

La culture musicale des Grecs et les barbares qui habitaient le littoral septentrional de la Mer Noire dans l'antiquité est encore peu étudiée. De plusieurs recherches archéologiques ont donné beaucoup de trouvailles, mais elles n'étaient pas interprétées comme il fallait. Notre travail vise à reconstituer quelques traits de la culture musicale des habitants de cette région, en nous appuyant sur les auteurs antiques et sur les trouvailles archéologiques. Nos tâches concrètes ont abouti à révéler les traits caractéristiques et les particularités de la culture musicale des Hellènes - d'un côté, et des barbares (des Scythes et des Sarmates) d'un autre coté, et à déterminer les instruments de musique des habitants de cette partie de l'oecumène antique dans la vie quotidienne, dans les rites des religions, au théâtre.

Les recherches assez circonstanciées consacrées à la culture musicale de l'Antiquité, aux instruments antiques de musique sont faites par les savants américains et français. Marta Maas dans son oeuvre "Stringed Instruments of Ancient Greece" a examiné l'origine des instruments à cordes, la technologie de leur fabrication, les mythes et les cultes dont ces instruments sont liés de l'avis des anciens. Daniel Paquette $^{2}$ caractérise un autre aspect - l'utilisqtion des instruments de musique dans des différents domains de la vie de la société antique. Sûrement, c'est le matériel dont l'auteur travaille - les vases couverts de peintures représent les images des plusieurs scénes avec des instruments de musique.

A regret, il n'existe pas de recherches détaillés au sujet de la culture musicale des colonies grecques du littoral septentional de la Mer Noire. On peut trouver des renseignements laconiques dans un petit chapitre

\footnotetext{
${ }^{1}$ M. Maas, Stringed Instruments of Ancient Greece (Bethany Connecticut: Brevis Press 1989).
} 
consacré à la culture des villes antiques dans l'édition en peusieurs volumes « L'archêologie de l'URSS de la haute antiquité au Moyen Age $»{ }^{3}$ E.E. Fialko a recueilli de certains renseignements sur la culture musicale des Scythes dans la partie «L'Art des peuples de Scythie la Grande $»^{4}$ V. M. Basilov ${ }^{5}$ et T. M. Kouznetsova ${ }^{6}$ examinaient aussi les instruments de musique des Scythes.

Les écrivains grecs notent, à l'unanimité, l'indifférence des Scythes à la musique, leur goût peu développé. Hérodote qui a donné la description en détail de la vie quotidienne des Scythes, notait qui'ils évitaient des emprunts des coutumes étrangères. A titre d'exemple, il raconte l'histoire de la mort du sage scythe réputé Anacharsys (Herodt, IV, 76-80).

Une telle négation du culte des dieux étrangers et l'attitude négative envers les instruments de musique grecs était assez ferme chez les Scythes . Domitius Callictratus, au I siècle av. J.-C. revient à l'image du sage scythe - il met dans sa bouche une malédiction à la flûte (Domit. Callicr. Epist., 6).

Plutarque (v.50 - v.125) dans ses «Oeuvres morales » cite des sentances des rois et des générals, et y compris, du roi scythe Atéi qui a dit qu'il préfèrait l'hennissement du cheval au jeu du flûtiste Isméni (Plutarch. Moralia, At).

L'auter des scholies de «L'Analytique tardive» d'Aristote ${ }^{7}$ mentionne de nouveau le sage scythe Anacharsys qui expliquait l'absence des flûtistes chez les Scytes par l'absence de la vigne et de

\footnotetext{
${ }^{2}$ D. Paquette, L' instrument de musique dans la cèramique de la Gréce Antique. Etudes d'Organologie (Paris, 1984).

${ }^{3}$ M. M. Kobilina, 'Teatr, aktery, musikalniie instrumenti', Antichniie gosudarstva Severnogo Prichernomoria (Moscow: Nauka1983) 219, Pl. CXIX.

${ }^{4}$ E. E. Fialko, 'Iskusstvo narodov Skifii' Velikaia Skifiia (Kiev-Zaporozhie 2002) 127139.

${ }^{5}$ V. N. Basilov, 'Skifskaia arfa' Sovetskaia etnografiia 4 (1991) 140-154.

${ }^{6}$ T. M. Kuznetsova, 'Vostochniie muzikalnije zerkala' Peterburgskii arkheologicheskii vestnik 7 (St. Petersburg 2000) 82-87.

${ }^{7}$ V. V. Latishev, Izvestiia drevnikh pisatelei o Skifii I Kavkaze (St. Petersburg 19921993) 147.
} 
l'ivrognerie (probablement, les Scythes comme et les Grecs, pensaient que le jeu de la flûte était lié étroitement avec la consommation du vin pendant les festins - symposiums collectifs et d'autres rites grecs liés avec Dionysos).

Il en résulte que les Scythes n'avaient pas absolument de musique, de chant, de jeu des instruments de musique. Est-ce possible? Plutarque dans les «Vies parallèles », à la biographie de Démétrios I ${ }^{\mathrm{er}}$ Poliorcète mentionne d'une coutume scythe étrange de pincer les cordes des ares pendant les festins (Plutarch. Vitae, XIX). La corde d'arc tendue donne le son vibrant agréable - c'est un prototype de tous les instruments à cordes. Quant an chant, nous n'avons qu'une mention chez Aristote. Dans ses «Problèmes » en s'efforçant de trouver l'origine du mot grec «vó $\mu \mathrm{o}$ » il mentionne une coutume d'une des tribus scythes de chanter les textes des lois (Aristot. XIX, 28).

Julius Polydeucus, grammairien et sophiste du II s. écrit qu'un instrument de musique à 5 cordes était inventé par les Scythes. Il était lié par 2 courrois de cuir d'oeuvre, en qualité de plectre on utilisait les sabots de chèvre. Il disait encore que les Scythes utilisaient les os des

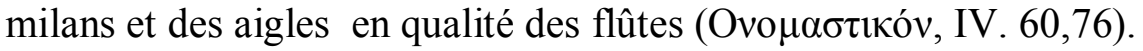

L'analyse des sources antiques nous persuade que selon les Grecs, l'art de musique était dévelopé faiblement chez les Scythes et des autres barbares du littoral septentrional de la Mer Noire. Mais les trouvailles archéologoques permettent de voir ce problème autrement. Les Scythes avaient ses propres instruments à cordes primitifs dejà à l'époque archaïque et à l'époque classique. Le plus ancien date VI s. av. J.-C.

Il est trouvé à l'Oural, dans un sépulcre du deuxième kourgane « Bachadarski ». C'est « une harpe scythe » - un instrument à cordes pincées, sa caisse était faite d'une morceau de bois, la membrane de cuir était collée au bois. Le support des cordes était mis à un trou special sur la caisse. ${ }^{8}$ Dans le sépulcre de femme riche du $8^{\text {ème }}$ kourgane

\footnotetext{
${ }^{8}$ S. I. Rudenko, Kultura naseleniia Tsentralnogo Altaia v skifskoie vremia (MoscowLeningrad 1960) 62-63.
} 
« Metchetsaïski ${ }^{9}$ on a trouvé l'instrument du type de la dombra. Dans le deuxième kourgane «Pazyrykski»» ${ }^{10}$ à Altaï , daté V s. av. J.-C. on a trouvé encore un instrument à cordes dont la réconstruction proposée à B. N. Basilov permet de voir le plus ancien instrument à archet du type $\mathrm{du}$ violin (fig.1). Son corps était fait d'un morceau de bois - il représente un billot vide rétreci au milieu, elle se fermait par une mémbrane fixée par des clous, au milieu était une table d'harmonie une petite planchette à un dessin cisélé . Les deux cordes étaient fixées sur le support des cordes et sur un manche. A l'aide de l'archet on tirait des sons. Cet instrument appartenait à un homme et le jeu avait une signification rituele. Un scythe jouant de l'instrument du type de la cithare (fig.2) est représenté sur une planchette d'or d'une coiffure du kourgane «Sakhnovski» (IV s. av. J.-C.). Les savants soulignent d'habitude le caractére rituel de cette scène. ${ }^{11}$ Ce qu'il y a de particulier c'est que l'action de culte est accompagnée du jeu de l'instrument de musique. Probablement, la femme tient à la main une glace-crécelle musicale qui donne le rythme à l'oeuvre interprétée. Principalement, c'étaient les Scythes - homme qui jouaient des instruments à cordes. Ainsi, "l'arrière violon" du deuxieme kourgane «Pazyrykski » mentioné si-dessus est trouvé dans le sépulcre d'homme. "La harpe » mentionée si-dessus est trouvée dans le sépulcre de couple de l'homme (du roi) et de la femme (de la concubine). Selon V. N. Basilov, cette coutume de l'appel au dieu à l'aide du jeu de l'instrument à cordes était le privilège du roi-sacrificateur et cette tradition est conservée jusqu'à present dans cette région chez les Kazakhs ce n'étaient que les chamans qui jouaient d'un instrument de musique pareil - « kobyz ». ${ }^{12}$ Plus tard, les Scythes utilisaient la lyre grecque pour ce but. Dans le crypte des Scythes № 9 (II-III ss.) de la nécropole de Neapolis de Scythie - capitale des Scythes, est représenté un homme jouant d'une lyre (fig.3). ${ }^{13}$ Dans

\footnotetext{
${ }^{9}$ K. F. Smirnov, Sarmati na Ileke (Moscow 1975)142-143.

${ }^{10}$ V. N. Basilov, 'Skifskaia arfa,' Sovetskaia etnografiia 4 (1991)140-154.

${ }^{11}$ S. S. Bessonova, D. S. Raevski, 'Zolota plastinka iz Sakhnivki,' Arkheologiia 21 (Kiev 1977) 30-50.

${ }^{12}$ Basilov, 'Skifskaia arfa,' 150-152.

${ }^{13}$ T . N. Visotskaia, Neapol-stolitsa gosudarstva pozdnikh skifov (Kiev 1979) 183.
} 
les tombeaux scythes et sarmates on a trouvé des glaces-crécelles musicales, 2 - à Altaï dans le $2{ }^{\text {ème }}$ kourgane «Pazyrykski » et dans le 5 ème kourgane «Rogozinski », et 1 - à l'Oural, dans le $8^{\text {ème }}$ kourgane «Metchetsaïski» (fig.4). ${ }^{14}$ Une telle glace représentait un disque rond composé de deux moitiés liées avec une queue. Une moitié était absolument lisse et servait de glase, une autre moitié avait une convexité et elle était remplie de petites goutes d'étain qui rendaient de beaux sons mélodiques à l'ébranlement. Ces glaces sont trouvées dans les sépulcres des femmes. Les femmes enterrée étaient danseuses et musiciennes. Ce fait est prouvé par ce que une enterrée dans ce $8^{\text {ème }}$ kourgane « Méchétsaïski» avait aussi un instrument du type de la dombra. ${ }^{15}$ Il faut noter la popularité des crécelles, des hochets, des clochettes qui rendaient un bruit rythmique musicale. Les Scythes aimaient faire une promenade " avec une clochette», aux decorations de fête des chevaux scythes on trouve des parures du cou avec des clochettes de cuivre et des pendeloques. ${ }^{16}$ On trouve des clochettes et dans les sepulcres. ${ }^{17}$ Ce sont des apotropées qui chassaient des esprits malins. Sur les bouts des bâtons rituels suspendait aussi des clochettes, en dedans on mettait une bille de fer (fig. 5). ${ }^{18}$ On peut supposer l'existence des crécelles fabriquées de l'astragals, des vertébres des animaux - on les trouve à l'abondance aux cités et dans les tombeaux des Scythes. ${ }^{19}$

La musique jouait un grand rôle dans la vie des Grecs anciens ils pensaient que la musique, la poésie et la danse exprimaient les sentiments de l'homme à l'aide des sons, des gestes et des mots. Elles

14 T. M. Kuznetsova, 'Vostochniie muzikalnije zerkala,' Peterburgskii arkheologicheskii vestnik 7 (St Peterburg 2000) 82-87.

${ }^{15}$ Smirnov, Sarmati na Ileke, 142-143.

${ }^{16}$ A. I. Meliukova, Krasnokutskii kurgan (Moscow 1981) 50-53, fig. 14.

${ }^{17}$ E. V. Chernenko, S. S. Bessonova, Yu. V. Boltrik, S. P. Polin, S. A. Skorii, Bokii N. M., Grebennikov Yu. S., Skifskie pogrebalnie pamiatniki stepei Severnogo Prichernomoria (Kiev 1986) 221, 264.

18 L. K. Galanina, Skifskiie drevnosti Podneprovia .Ermitazhnaia kollektsiia N. E. Brandenburga. SAI. D1-33 (Moscow 1977) 41-42, P1.21.

${ }^{19}$ Chernenko et als., Skifskie pogrebalnie pamiatniki stepei Severnogo Prichernomoria, 216-217, 222, 238, 270, 279. 
serraient à adoucir et à calmer les sentiments, à purifier le coeur (catharsys). La musique s'est separée de celte chorée (cette triade)- selon T. Zelinski - et s'est appropriée sa fonction dominante de l'art expressif - servir à exprimer les sentiments. ${ }^{20}$ Avec cela, elle conservait la liason avec la culte et la réligion: on chantait le péan à l'honneur d'Apollon, le dithyrambe - à l'honneur de Dionysos, les prosodies - pendant les processions. La musique était une partie des mystères, on l'estimait comme le don des Dieux, on l'attribuait des caractéristiques particulières, une force magique, lui était naturel de charmer, d'enlever la latitude de l'homme et des animaux. Les sectes orphiques pensaient que la musique pouvait libérer l'esprit du corps pour un certain temps.

La culture musicale des villes-colonies grecques du littoral septentrional de la Mer Noire était très élevée. A Khérsonèse, on a fouillé le théâtre antique unique sur le littoral Nord-Pontique (mais les sources anoncent qu'il y avait encore des théâtres dans les autres villes de cette région).Aux théâtres on mettait en scéne des tragédies et des comédies ce que attestent les inscriptions et les images des acteurs et les masques comiques et tragiques de terre cuite, dont la plupart est faite dans cette région. Sauf les représentation, aux théâtres y avaient lieu les concours de musique et littéraires, par excellence, à l'époque hellénistique. A Khérsonèse s'est conservée l'inscription du catalogue des concours de musique. Les maîtru existaient aussi - les musiciens professionnels. ${ }^{21}$ Le musicien et poète de Bosphore Isill (à la première moitié du III s. av. J.-C.) participait aux concours de musique à Dêlphes. ${ }^{22}$ A Mirmécée on a trouvé une épitaphe du flûtiste Pasaphilicatos (IV s. av.J.-C). ${ }^{23} \mathrm{Au}$ cours du fouilles on a trouvé beaucoup d'artéfacts qui prouvent une large diffusion de différents instruments de musique dans la vie quotidienne des habitants - on a

\footnotetext{
${ }^{20}$ V. Tatarkevich, Antichnaia estetika (Moscow 1977) 17.

${ }^{21}$ E. I. Solomonik, Noviie epigraficheskiie pakiatniki Khersonesa. Lapidarniie nadpisi (Kiev 1973) 99, 103.

${ }^{22}$ E. B. Petrova, Antichnaia Feodosiia (Simferopol 2000) 156.

${ }^{23}$ Kobilina, M. M. 'Teatr, aktery, musikalniie instrumenti', Antichniie gosudarstva Severnogo Prichernomoria (Moscow: Nauka1983) 219.
} 
fouillé les fragments de la flûte, de la ryre, les terres cuites avec les images de la syrinx, de l'harpe, de la cithare, du tympanon. La plus grande partie de l'information nous donnent les images sur les vases couverts de peinture fouillé dans les cité du littoral septentrionales de la Mer Noire. Elles nous représentent les goût d'art de la population indigène. L'abondance des images des scénes avec des musicien étonnece sont des danses avec accompagnement musical, des scènes de théâtre, des festins, des loisirs. Un tel choix des sujets de la peinture s'avère par des documents antiques sur l'amour des Grecs à la musique, au chant et aux danses.

Les instruments de musique des Grecs étaient simples, leur son n'était pas fort, ils ne permettaient pas de montrer la maestria, on les utilisait pour accompagner au chant et à la danse. On connait les instruments à cordes suivants: la lyre, la cithare, la harpe ; les instruments à vent : la flûte, la syrinx, la salpinx, le kéras ; on utilisait les instruments à percussion (le tympanon, les crotales) pendant la danse pour donner le rythme. D'ordinaire le rythme predominait sur la mélodie dans la musique grecque. Dionysos de Halicarnasse ecrivait que les mélodies charmaient l'oreille et le rythme l'excitait. ${ }^{24}$

L'instrument à vent le plus courant est l'aulos-chalumeau hellénique nous rencontrons le plus souvent sur nos vases. L'aulos simple est coustitué par un tube de perce cylindrique ou conique, muni d'un embout où se fixe l'anche et de deux ou trois éléments mobiles en forme d'olive. ${ }^{25}$ Un pavillon le complète parfois. L'instrument le plus curant est l'aulos double (diaulos), composé d'un double chalumeau dont les tuyaux, distincts et complémentaires, sont sonnés simultanément, les anches enfoncées complètement dans la bouche, et chaque main agissant sur l'un d'eux. C'est par les mouvements des tubes dans l'espace qu'on obtenait les ressources sonores que procure notre système de clefs. Sur les vases des $\mathrm{VI}^{\mathrm{e}}$ et $\mathrm{V}^{\mathrm{e}}$ siècles, les tuyaux apparaisent en teinte claire. Il est logique de penser que le bois, facile à travailler, propre à fournir une bonne réponse acoustique, était couramment utilisé. Le roseau, le

${ }^{24}$ Tatarkevich, Antichnaia estetika, 19.

${ }^{25}$ S. Gazarian, V mire muzikalnikh instrumentov (Moscow 1989) 49. 
sureau, le lotus, le buis étaient les essences d'arbres dont les facteurs d'instruments se servaient pour tourner les tubes, avec une préférence probable pour le buis. Le cerisier, prunier et l'erable ont aussi de bonnes qualités pour la perce des tubes. Le métal ou l'ivoire plus coûteux, l'os plus difficile à façonner, ont sans doute été des matériqux plus rarement utilisés (un fragment d'un aulos d'ivoire est fouillé à Panticapée). ${ }^{26}$

La taille des tuyaux varie d'un vase à l'autre. Ces différences attestent l'existence d'une véritable famille d'aulos, répondant aux cinq tessiture de la voix humaine. En calculant les dimension approximatives des instruments d'après la taille des personnages sur les vases, on peut établir la classification ${ }^{27}$ suivante :

a) soprano (de 20 à $30 \mathrm{~cm}$ )

b) alto (de 40 à $50 \mathrm{~cm}$ )

c) ténor (de 50 à 80 )

B) baryton (de 80 à $1 \mathrm{~m})$

c) basse (1 m et plus)

Selon les trouvailles archéologiques, la taille moyenne se situe autour de $40 \mathrm{~cm}$, sur les vases nous voyons plus souvent l'alto, le ténor et la baryton (fragment d'un cratère du musée de Khérsonèse № 2877/09) (fig. 6), un cratère du musée historique de Kiev avec l'image d'une scène théâtrale (fig. 7), une péliké du musée de Jalta (№ 502) (fig. 8). Sur de nombreux vases, les tuyaux sont dessinés égaux: ainsi l'aulète pouvait, de les doits posés à plat, boucher en même hauteur, sur les deux tubes placés en parallèle. A ce cas on faisait l'effet de la stéréophonie. Si on bouchait les trous situés à l'hauteur différent, on produisait l'intervalle. Nous ne voyons presque pas des aulos à tubes inégaux sur les vases - par exemple un basse -baryton, ets.

L'instrument à cordes le plus courant était la lyre. A la différence de la cithare lourde, dont il fallait tenir verticalement et celui qui joue de la cithare devait être débout, la lyre plus légère permettait à la pencher

${ }^{26}$ Kobilina, M. M. 'Teatr, aktery, musikalniie instrumenti', Antichniie gosudarstva Severnogo Prichernomoria (Moscow: Nauka1983) pl. CXIX, 8.

${ }^{27}$ Paquette, L' instrument de musique dans la cèramique de la Gréce Antique, 25-26. 
pendant le jeu, danser avec elle sans arrêter à jouer la mélodie. On tirait le son à l'aide d'un plectre - une lame forte de fer ou d'os. Dans la plupart des cas, la main droite tient le plectre, les doigts de la main gauche bloquaient certains cordes pour émettre des sons différents. Primordialement, c'était la carapace d'écaille qui servait du résonateur. Elle était couverte de cuir. Les bras (à l'origine cornes de bovidés ou d'ovides) et la traverse tenaient et tendaient les cordes.

Les cordes etaient en nombre variable - de 5 à 9 . Au bas de la carapace il y avait des trous pour les cordes qui se roulaient sur la traverse. Une lyre à 5 cordes est représentée sur une péliké du musée de Yalta № 502 (fig. 8). Sur ce dessin elle est représentée de l'intérieur, sur une pyxis de même musée №496 (fig. 9) - de l'extérieur de l'instrument. Eros qui tient la lyre à la main gauche sur ce dessin, tend le plectre à la main droite à une femme. Eros, dieu de l'amour, est souvent représenté avec une lyre à la main quand il observait les préparatifs de noces (comme sur cette pyxis). Mais le dessin sur la péliké représente Eros avec une lyre et une des Muses.

La cithare, instrument d'Apollon et des virtuoses de concours, le plus perfectionné et sans doute le plus complet de la Grèce classique. La résonance considerable due à une caisse de grande dimension, à la forte tension des cordes, peut-être à des lames vibrantes, favorisait son emploi « public ». La tessiture devait se situer au niveau du violoncelle actuell, ce qui lui confère un ambitus de baryton, favorable à l'accompanement des vois virilis. La lyre et la cithare étaient tres populaires chez les habitants du littoral septentrional de la Mer Noire (fig. 10). Nous en avons un témoignage concluant de Poliaenus (II s.). Il reconte d'une action de reconnaissance de Philippe de Macédoine Memnon qui recourait à l'aide du citharède Aristonic à une reconnaissance. Ce fragment atteste un grand intérèt des habitants des villes de Bosphore à une célébrité de passage, leur amour de la musique (Poliaen. Strategemata. V, 44).

Un des instruments à percussion les plus répandus que nous pouvons voir sur les vases des musées de Crimée est le tympanon. Il est constitué d'une peau tendue sur un cercle de bois dont le diamètre peut 
être varié de $30 \mathrm{~cm}$ - à $80-90 \mathrm{~cm}$. On peut le voir sur les plusieurs images sur les vases (fig.10) - parfois il a la taille d'un bouclier et il couvre tout le haut du corps humain, parfois il a la taile d'une petite assiette. Le tympanon est utilisé essentiellement pendant les danses et cérémonies orgiastiques, les rites de Dionysos. On venait avec lui aux tombeaux des parents et on chantait à mi-voix. Il accompagnait les mystères, on voit souvent les figures des mystos aux bondeaux avec les tympanons à la main.

Les Grecs ont connu la trompette - salpinx sous la forme la plus simple: tube droit terminé d'un côté par un pavillon, de l'autre - par une embouchure(fig.11). La salpinx pouvait mesurer de $60 \mathrm{~cm}$ à $120 \mathrm{~cm}$, elle n'avait jamais de perforations. ${ }^{28}$ La salpinx possédait un pavillon de forme variée :en boule, en bulbe, en cône . La salpinx avait une tessiture voisine de celle de notre violon alto et sonnait dans un registre médium aigu. La trompette est normalement faite en bronze, mais il existait aussi des trompettes de fer. La salpinx parait avoir été relativement légère car l'instrumentiste la tient d'une seule main. Au ses faibles possibilités musicals et le caractère perçant de sa sonorité, la trompette est essentielement un instrument d'alerte ou de signalisation. Dans la vie militaire, elle donne le signal de l'attaque ou de la retraite. Au théâtre, elle sert à obtenir le silence. Sur les vases, elle aparait entre les mains des Amazones ou de Niké. Un épisode très interessant de l'histoire de Théodosie antique est lié avec cet instrument. Le général Tinnikh à l'aide de la salpinx a liberé cette ville du siège [Poliaen. Strategemata.V, 23].

L'instrument de signaux plus primitif était fait de coquille du mollusque de mer - un tel exemplaire est trouveé pendant les fouilles à Kalos- Limène (colonie de Khérsonèse de Tauride à la Crimée de sudouest). ${ }^{29}$

L'instrument était trouvé dans les couches de la destruction liées avec la dernier siège de la ville par les Scythes, entouré des artéfacts temoignant que c'était un outillage d'un navire de guerre tiré sur la côte.

${ }^{28}$ Paquette, L' instrument de musique dans la cèramique de la Grèce Antique, 74 -75.

${ }^{29}$ Communication aimable de V. Ougentsev. 
Nous pouvons voir une image d'une harpe ou d'un trigonon sur les statuettes de terre cuite de Panticapée(d.12). Une syrinx - l'instrument préféré de Pan - est représenté sur les terres cuites de Panticapée(id.13).

Donc les sourses antiques, les trouivailles archéologiques et les images sur les vases prouvent que les habitants des villes antiques du littoral septemtrional de la Mer Noire utilisaient à la vie quotidienne, à l'activité professiolelle, pendant les rites réligieux utilisaient les instruments à cordes. On connait la lyre, la cithare,la harpe ou le triginon. Parmi les instruments à vent on connait la flûte,la syrinx et la trompettesalpinx. L'instrument à percusson le plus répandu est tympanon.

Sans aucun doute qu'ils etaient faits dans les atelier d'artisan au littoral septembrional de la Mer Noire, et leurs sons réjouaient l'oreille des melomanes de cette régione dont il y avait beaucoup(selon les auteurs antiques).

Les Scythes et les Sarmates préféraient les instruments à cordes faits des matériaux de fortune de bois, de peau, des tendons des animaux. Les orecelles des hochets étaient importés et fabriqués à la region. Les ornements, les vêtements feminins de fête abondaient en pendeloques faisant $\mathrm{du}$ bruit aui pouvaient accompagner à une danse. Le harnochement produisait aussi des sons melodieux grâce aux sonnettes pendues. La musique accompagnait les rites, on jouait de l'instrument à cordes, et, probablement, une sacrificatrice qui donnait le rythme à l'aide d'une glace musicale ou d'une crecelle. Le départ d'une armée était accompagné de divers bruits musicaux. Les pommes des cannes qui ornaient les bâtons de rite, faisaient de bruit. Un aristocrate scythe au cheval représentait un phénomène non seulement majestueux et redoutable, mais aussi et musical grace aux sonnettes et les autres pendentifs sur le harnachement. Les flèches des Scythes sifflaient au vol. La musique accompagnait et les festins, et la vie quobidienne, et la loisir.

Pour faire notre notion de la culture musicale des peuples indigènes plus détaille, il faut réunir toutes sortes des sourses - et les renseignements des auteurs antiques, et les trouvailles archéologiques les débris des instruments de musique et les images des scènes avec les 
instruments de musique sur les vases, sur les terres cuites, sur les monuments trouvés dans cette région.

La création d'un tel corps - notre tache immédiate. Nous espérons que notre travail soit utile aux critiques d'art, aux musicologues et au milieu large des amateurs de l'Antiquité .

\section{Figures:}

Fig. 1. Instrument à archet du type du violon trouvé dans le deuxième kourgane «Pazyrykski » à Altaï (V s. av. J.-C.)

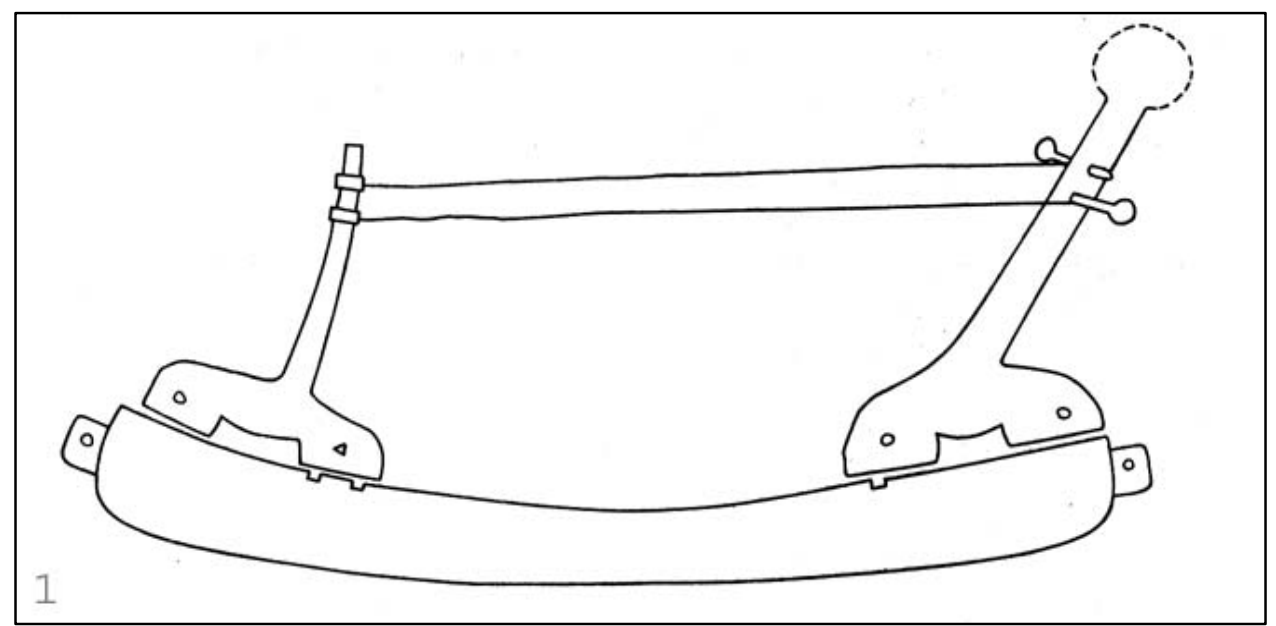

Fig.2 : Un Scythe jouant de l'instrument à cordes du type de la cithare image sur la planchette d'or d'une coiffure trouvée dans le kourgane "Sakhnovski" (IV s. av. J.-C.). Kiev. Musée des objets précieux historiques.

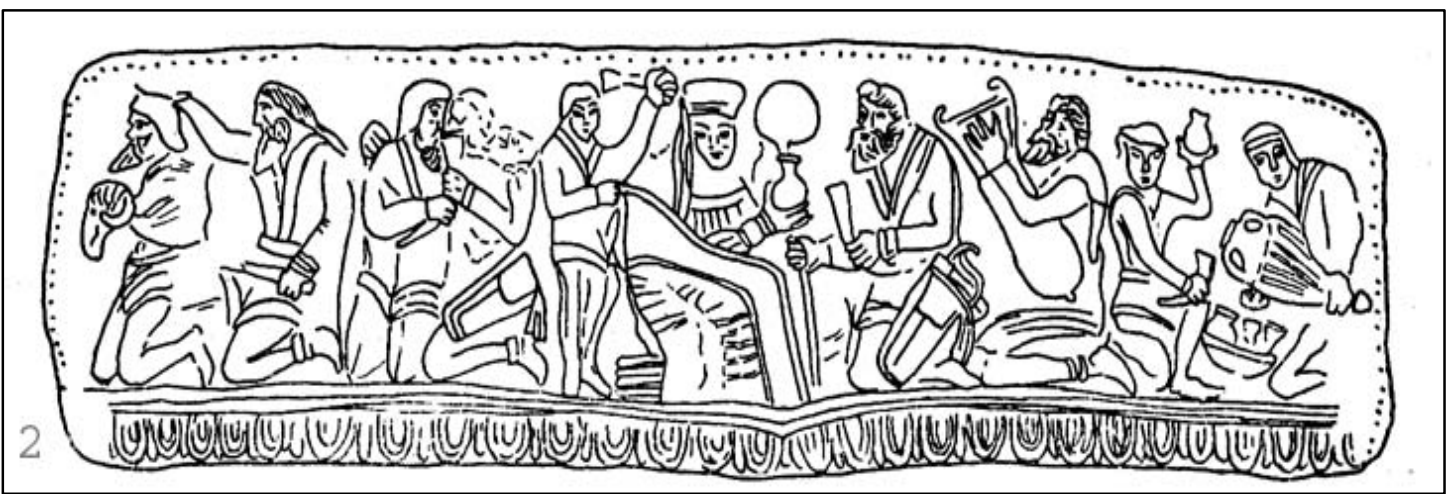



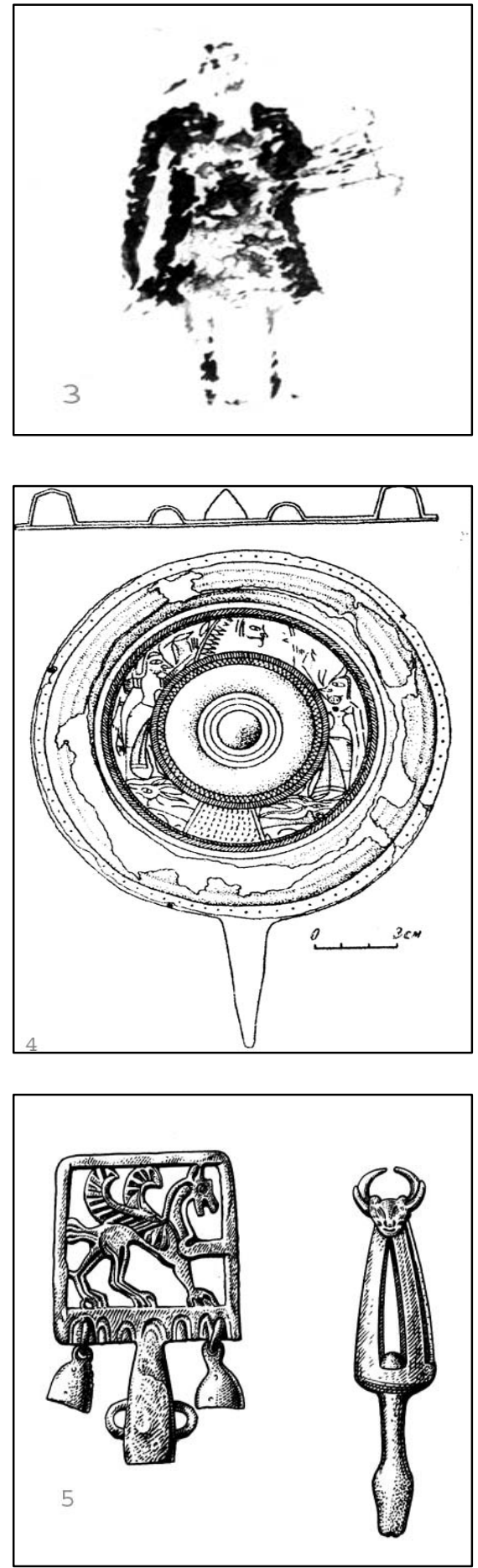

Fig. 3 : Un homme jouant de la lyre. Caveau №9 (II-III ss.) de la nécropole de Néapolis de Scythie capitale des Scythes tardifs.

Fig. 4: Une glace-crécelle musicale trouvée dans le sépulcre sarmate « Metchetsaïski »(kourgane8,enterre ment5).D'après K.F. Smirnov

Fig.5 : Les bouts des bâtons rituels scytes:

a) kourgane «Alexandropolski»

b) kourgane «Starchaïa moguila» 


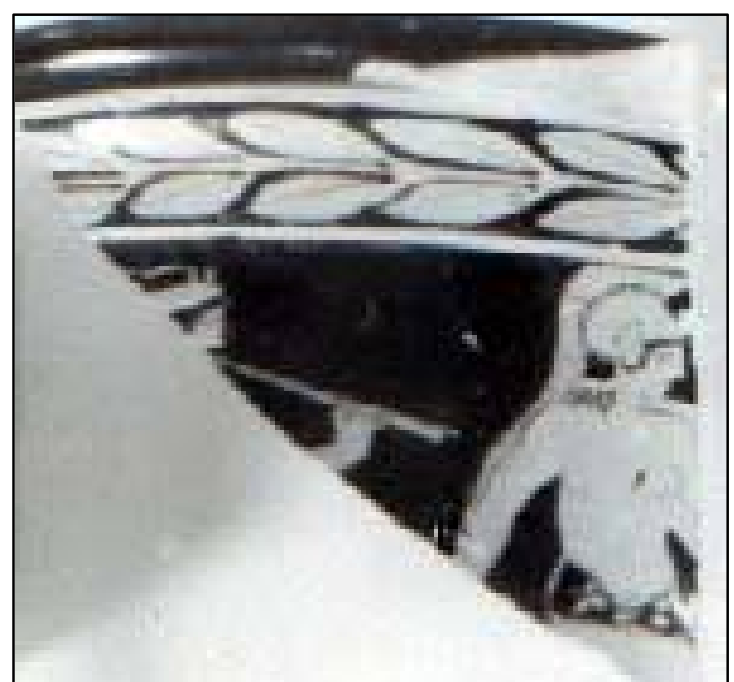

Fig. 6: Aulos-alto. Fragment du cratère du musée de Khérsonèse (№ KP - 2877/08)

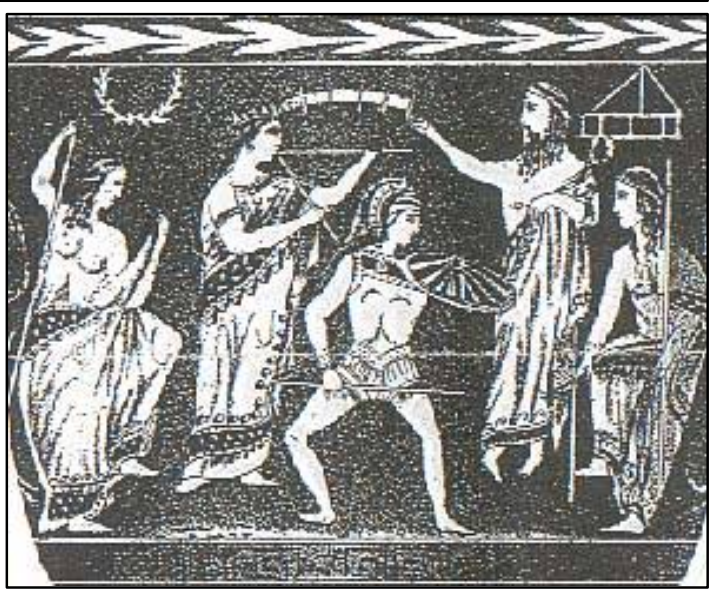

Fig. 7 : Aulos-baryton. Cratère avec l'image d'une scène théâtrale. Musée historique de Kiev.

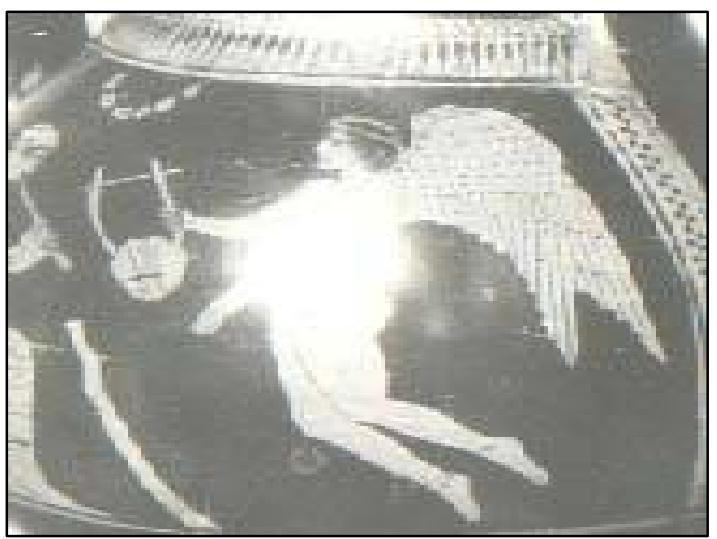

Fig.8: Aulos-soprano. Péliké du musée de Yalta, KP - 502. Ici il y a encore une image qui représente une lyre à 5 cordes à l'intérieur. 


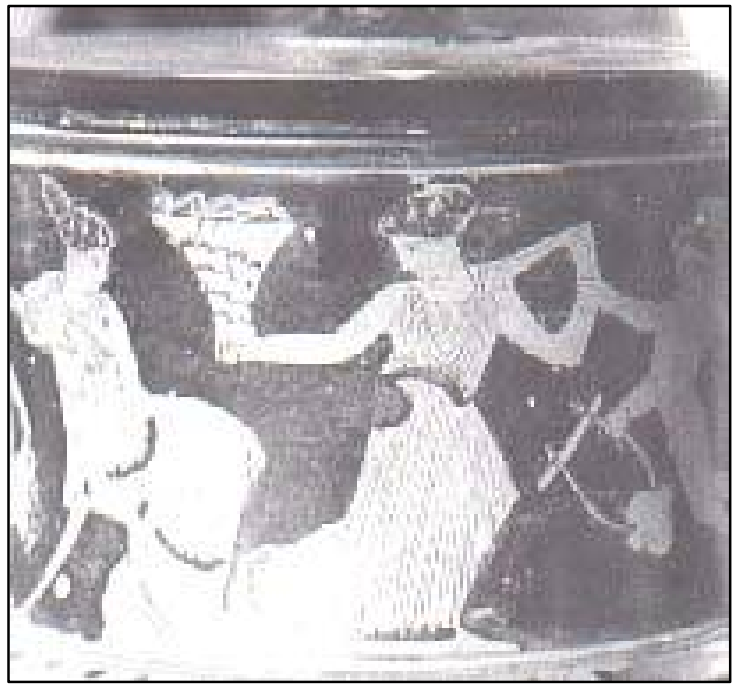

Fig.9: Lyre sur une pyxis de musée de Yalta (KP - 496).

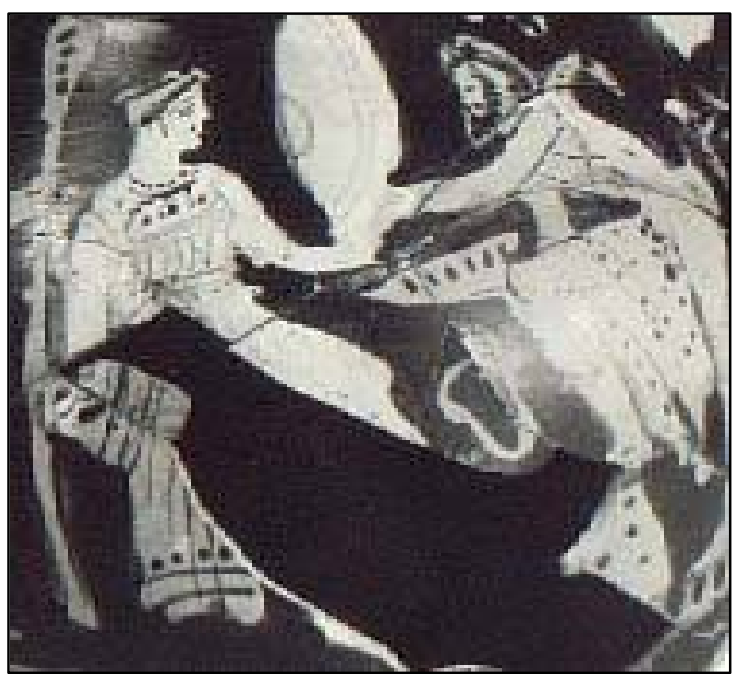

Fig. 10: Image d'un tympanon sur une péliké du musée républicain de Crimée (KP - 1094).

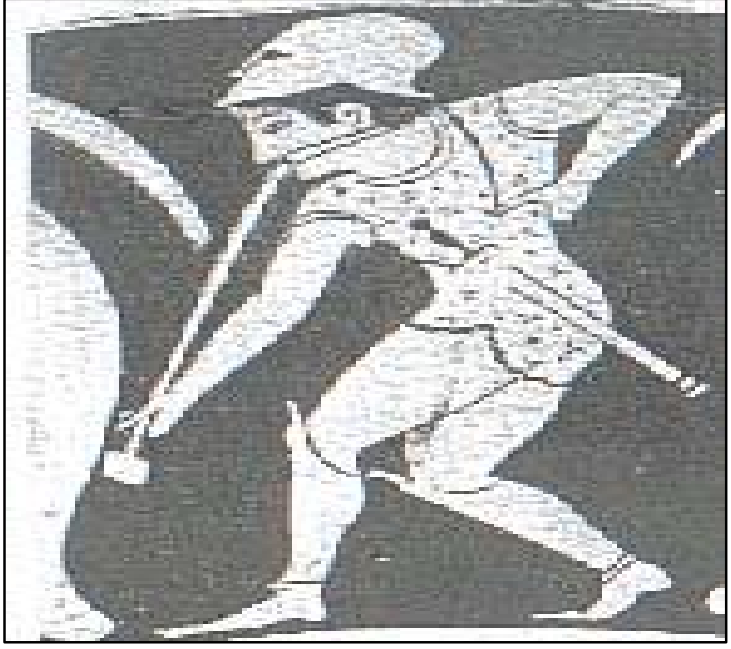

Fig. 11.

Salpinx..Coupe,FR,

Vatican n ${ }^{\circ} 498$, ARV $^{2}$ 


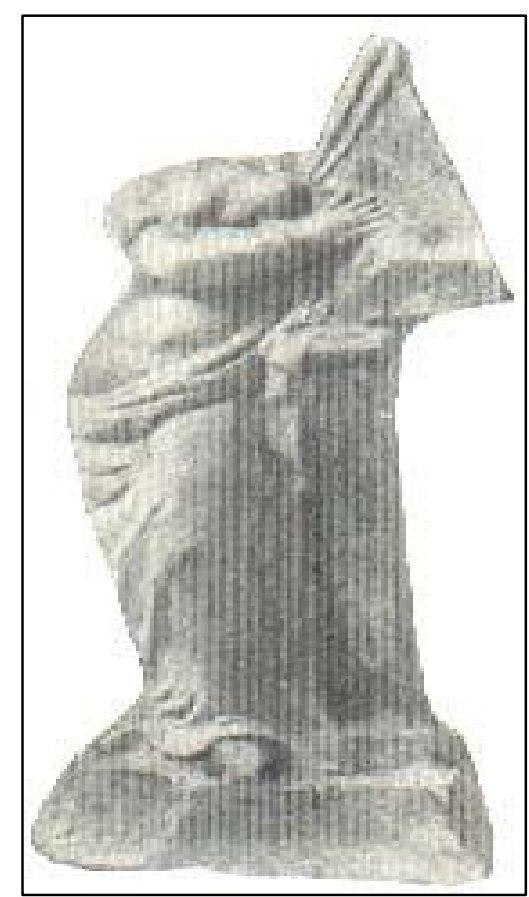

Fig. 12: Harpe ou trigonon sur la statuette de terre cuite de Panticapée.

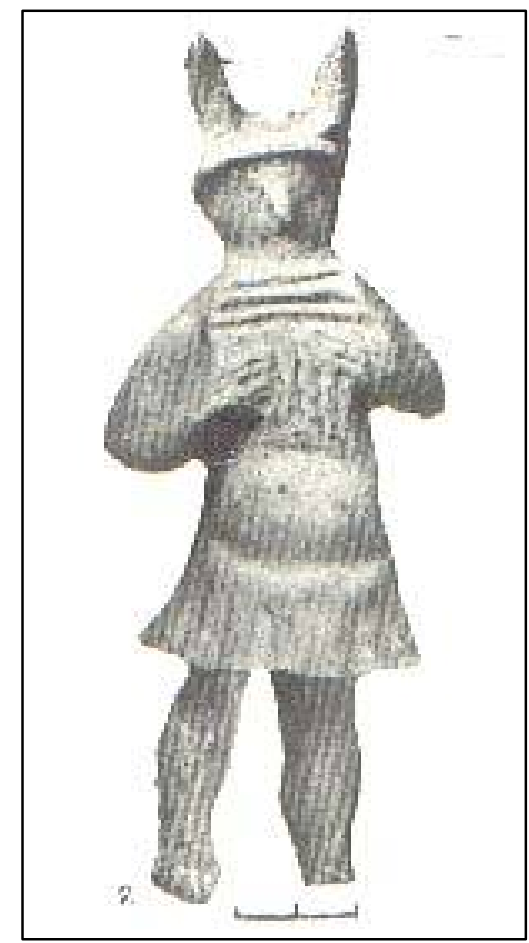

Fig. 13: Syrinx sur une terre cuite de Panticapée. 